11

\title{
Исследования воздействия радиочастотного и ионизирующего излучений на микроконтроллер ATmega8515
}

\author{
() А.П. Степовик, Е.Ю. Шамаев, В.В. Отставнов
}

Российский фредеральный ядерный центр - Всероссийский научно-исследовательский институт технической фризики им. акад. Е.И. Забабахина,

456770 Снежинск, Челябинская обл., Россия

e-mail: dep5@vniitf.ru

(Поступило в Редакцию 2 марта 2018 г.)

Представлены результаты исследований действия сверхширокополосного и тормозного излучений на сложно-функциональное устройство типа ATmega8515 в радиопрозрачном корпусе. Показано, что реакция микроконтроллера на облучение в обоих случаях близкая: реализуется кратковременный сбой в работе или зависание. Реализация эффектов зависит от момента действия излучений относительно фазы генерируемого микроконтроллером меандра. В то же время результат облучения тормозным излучением зависит и от экспозиционной дозы, что может приводить как к уширению генерируемой фазы сигнала, так и к зависанию устройства.

DOI: 10.21883/JTF.2019.03.47183.153-18

\section{Введение}

В настоящее время в современной радиоэлектронной аппаратуре (РЭА) используются сложные устройства на основе новых перспективных компонент микроэлектроники, в частности больших (БИС) и сверхбольших (СБИС) интегральных микросхем. Исследования физических процессов в подобных устройствах при действии ионизирующего излучения (ИИ) проводятся уже не одно десятилетие. Однако в последние годы одной из насущных проблем стала проблема их устойчивости к действию радиочастотных излучений: СВЧ, сверхкоротких (СК), сверхширокополосных (СШП) импульсов электромагнитного излучения (ЭМИ). Ее появление связано как с потенциальной террористической угрозой, так и с возможной разработкой радиочастотного оружия. На эту тему опубликовано большое количество работ, в которых показана опасность действия СШП ЭМИ, например, [1-3]. Как было получено авторами, реакция одного из сложно-функциональных компонентов - микроконтроллера (МК) на их действие заключается в появлении различного рода сбоев в работе, от однократного с самовосстановлением до так называемого „зависания“, когда для восстановления работоспособности необходимо вмешательство оператора.

Не исключено, что одни и те же компоненты микроэлектроники могут применяться как в условиях действия ИИ, так и при действии радиочастотного излучения. Отсюда представляет интерес рассмотрение их поведения в условиях раздельного действия этих факторов.

\section{1. Методические вопросы проведения исследований}

Для исследований был выбран МК широкого применения в радиопрозрачном корпусе ATmega8515. Такие устройства обладают свойствами миникомпьютера, и они являются основой блоков управления большого количества электронных систем. Отсюда сбой функционирования МК может привести к сбою или отказу в работе этих систем. Последствия реализации такого сценария могут быть самыми разными: от необходимости перезапуска системы до катастрофического отказа, если система выполняла жизненно важные функции.

Выбор конкретного типа МК был обусловлен двумя факторами:

- полученными авторами данными о возможности сбоя в его работе при облучении пачками СК (длительность $0.1 \mathrm{~ns})$ СШП ЭМИ [4];

- имеющимися литературными данными о его радиационной стойкости (примерно $10 \mathrm{kR}$ ) к действию ИИ, полученными в условиях стационарного облучения на источнике $\mathrm{Cs}^{137}$ [5].

МК ATmega8515 состоит из большого количества функциональных блоков, которые условно можно разделить на несколько групп: вычислительное ядро, подсистема памяти, периферийные устройства, система тактирования, выходные буферы. В состав вычислительного ядра входят арифметико-логическое устройство (ALU), регистры текущего состояния и контроля, оперативнозапоминающее устройство (ОЗУ), регистры общего назначения и т.д. При подаче питания на МК происходит процедура инициализации ядра, включающая первоначальную настройку оборудования согласно параметрам изготовителя. Затем происходит загрузка программы, записанной во внутреннюю флэш-память.

В составе МК ATmega8515 содержится 5 портов, из которых 4 - стандартные восьмиразрядные, а один порт имеет 3 вывода. При проведении экспериментов использовали только порт $A$ (активный порт был сконфигурирован как выход), остальные находились в Z-состоянии. Для контроля функционирования МК была написана программа, которая выполняла инкремент значения 
регистра PORTA в бесконечном цикле [4]. Тактирование осуществлялось от внутреннего $R C$-генератора с частотой $8 \mathrm{MHz}$. Штатная работа МК характеризовалась периодической сменой логических состояний (период $\sim 1.2 \mu \mathrm{s}$ ), что на экране осциллографа регистрировали как меандр бесконечной длительности, а функциональный сбой - его прекращение на разный промежуток времени.

При облучении СШП ЭМИ использовали пачки из 100 импульсов, которые следовали в них с частотой $1 \mathrm{kHz}$, что связано с малой вероятностью сбоя работы МК одиночным импульсом. Облучение ИИ (тормозным излучением, ТИ) проводили одиночными импульсами на мощной импульсной электронной установке [6], поскольку такой режим является для нее рабочим. В этих условиях частота тактирования $8 \mathrm{MHz}$ была более предпочтительной: как было получено, вероятность сбоя МК данного типа увеличивается с ростом частоты.

\section{2. Облучение СШП ЭМИ}

Экспериментальные исследования были проведены в безэховой камере с использованием генератора ЭМИ СВЧ-диапазона на основе сверхсветового источника $[7,8]$ с длительностью излучения на уровне $\sim 10^{-10} \mathrm{~s}$ и напряжением на генерирующем фотодиоде $(Ф Д) \sim 64 \mathrm{kV}$. При таком напряжении соответствующий диапазон излучаемых частот составляет примерно 1-30 GHz. Вспомогательное оборудование (источник питания МК - аккумуляторная батарея и линейный стабилизатор) разместили в экранированном прямоугольном корпусе. МК закрепили на кронштейне (стальной трубке), вмонтированном в одну из граней корпуса (рис. 1). Через трубку проходили линии связи для передачи информации на осциллограф и провода для подачи на него питания.

Ориентация экранирующего корпуса под углом $45^{\circ}$ к направлению падения излучения обеспечивала отсутствие влияния его отражения от корпуса на тыльную сторону МК. Для измерений амплитуды напряженности электрического поля использовали датчик ИППЛ-Л [9]. Диапазон изменения напряженности в месте расположения МК $65-180 \mathrm{kV} / \mathrm{m}$, погрешность ее измерений обу-

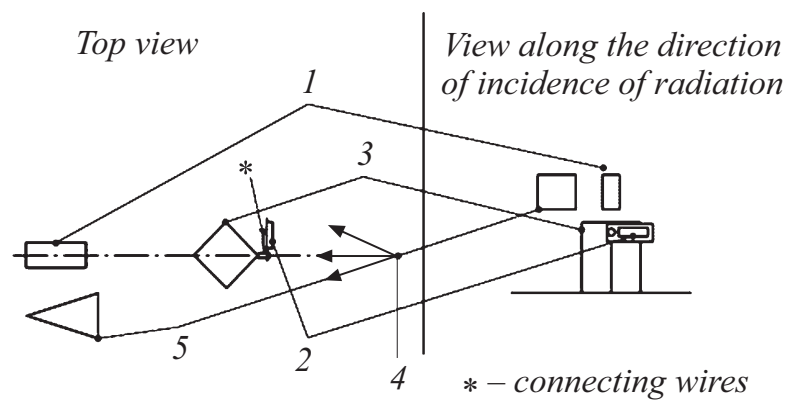

Рис. 1. Схема проведения экспериментов с СШП ЭМИ: 1 датчик ИППЛ-Л, $2-$ МК, 3 - корпус устройства, $4-$ ФД, 5 - рупорная антенна П6-123А. словлена в основном погрешностью калибровки датчика $( \pm 10 \%)$, относительная погрешность $1-3 \%$.

Ввиду большой скважности СК-импульсов $\left(\sim 10^{7}\right)$, одновременное отображение на развертке осциллографа момента действия излучения и меандра достигалось с помощью интегрирующей схемы, используемой персоналом источника для относительных измерений их амплитуды при следовании с частотой $1 \mathrm{kHz}$. Схема состоит из рупорной антенны П6-123А для приема излучения и интегрирующей цепи с входным резистором $1 \mathrm{M} \Omega$ на входе цифрового осциллографа типа Tektronix. После каждого облучения осциллограммы записывались в стандартные для данного типа осциллографа файлы с расширением wfm.

МК облучали в состоянии поставки, внешние выводы перпендикулярны его корпусу и вектору электрической компоненты поля, большая сторона корпуса параллельна ему. В результате излучение принималось только его внутренними дорожками [4]. С целью повышения статистической достоверности результатов количество повторений облучений в одинаковых условиях составляло 100 раз.

На рис. 2 приведены осциллограммы некоторых наблюдаемых видов сбоя. Термином „зависание“ обозначили такой вид, при котором восстановление периодической смены состояний, наблюдаемое на длительности развертки осциллографа, после окончания действия пачки импульсов не происходило. Термином „ $\delta$-сбой“ обозначили сбой с длительностью, меньшей $1 \mathrm{~ms}-$ периода следования импульсов в пачке. Как видно из рис. 2, наблюдается большое разнообразие сбоев МК разной длительности на протяжении действия пачки. Однако несмотря на это существуют только два кардинально различающихся вида сбоя: зависание и $\delta$-сбой, остальные отличаются только длительностью, которая может изменяться от единиц до десятков ms.

Были проведены исследования зависимости реализации различных эффектов в МК от величины напряженности поля, в частности, приведенных на (рис. 3).

На основании данных экспериментов было получено, что зависание является пороговым эффектом: оно реализуется в $\sim 100 \%$ случаев при однократном сбое и минимальной величине напряженности поля. В то же время уже относительно небольшое (на $\sim 10 \%$ ) увеличение напряженности поля приводит к росту вероятности возникновения многократного сбоя и в результате возможности восстановления работы МК. По-видимому, при этих напряжннностях амплитуд электрических напряжений, наводимых на внутренних дорожках МК [4], достаточно, чтобы переход в него произошел, но самостоятельно, без вмешательства извне или работы заложенной программы или воздействия остальных импульсов пачки он из этого состояния выйти не может. Переход в зависание напоминает защелку тиристорный эффект в КМОП полевых транзисторах. Необходимо отметить, что данный эффект скорее всего связан с наведенными потенциалами на выводе „reset“ 

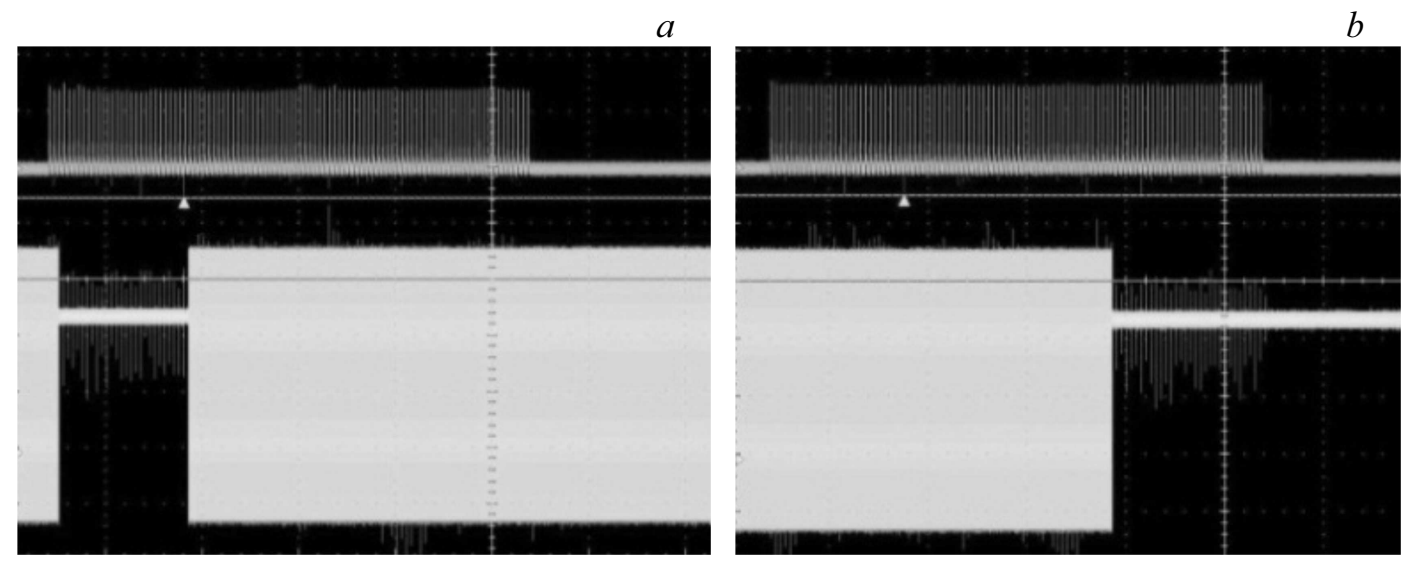

$c$
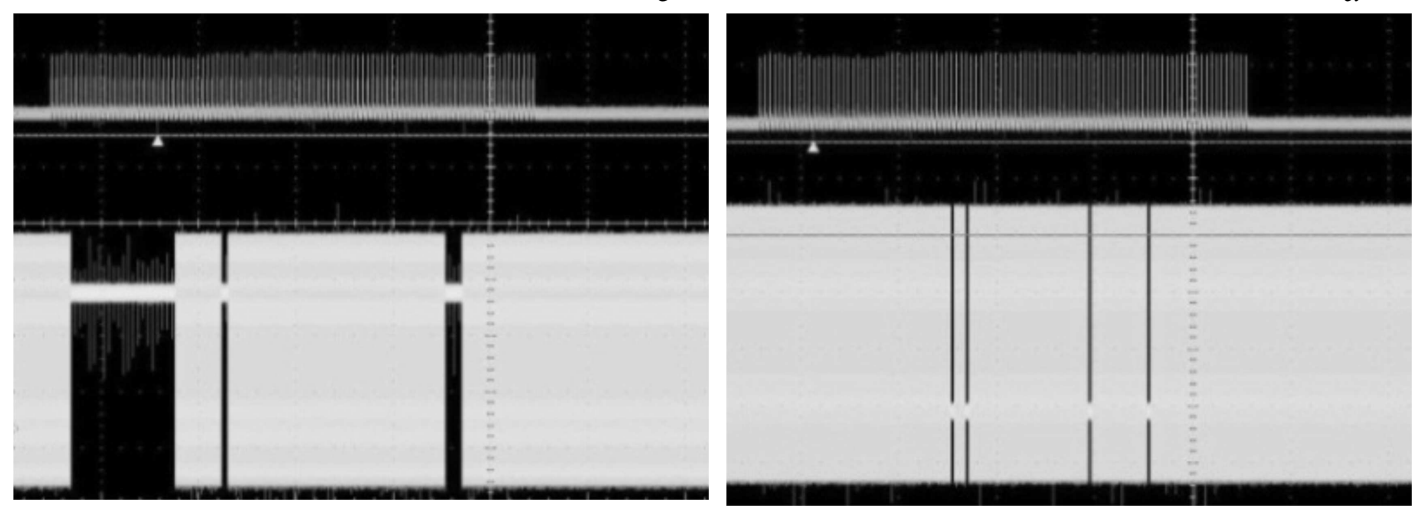

Рис. 2. Типичные виды зарегистрированных сбоев МК. Верхний луч - пачка импульсов излучения (длительность пачки 100 ms), нижний луч - меандр периодической смены логических состояний. $a-$ однократный с восстановлением; $b-$ однократный с зависанием; $c$ - многократный с восстановлением; $d-$ многократные $\delta$-сбои с восстановлением.

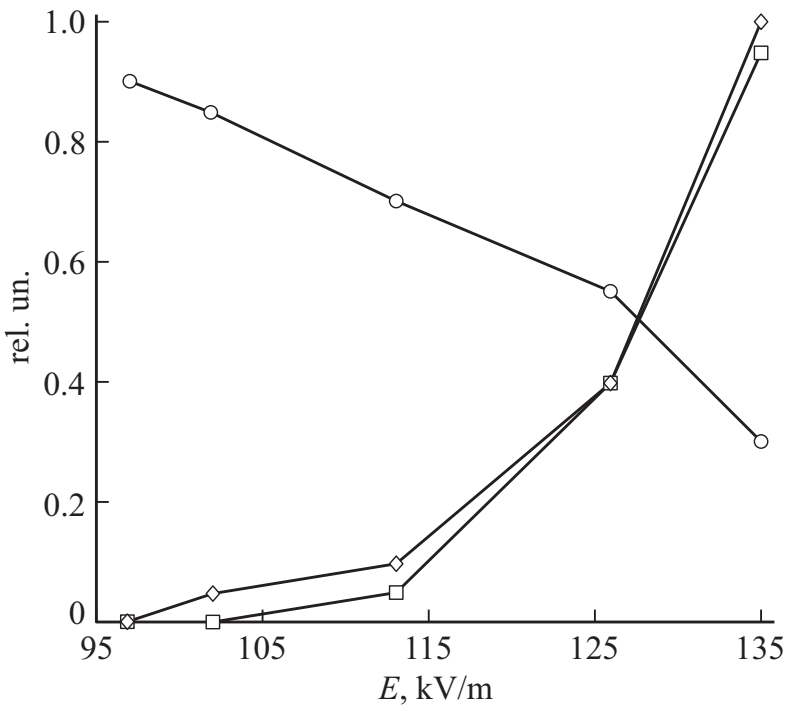

Рис. 3. Изменение долей некоторых видов сбоев МК в общей их совокупности в зависимости от напряженности поля. одоля зависаний, $\square-$ доля $\delta$-сбоев, $\diamond-$ доля мноугократных сбоев.

и не связан с увеличением тока потребления, как при тиристорном эффекте.
Одной из основных задач исследований являлось выяснение условий, необходимых для реализации сбоя в работе МК. Было предположено, что одним из условий может быть существование определенных временных соотношений между моментом действия излучения и фазой работы МК, когда действие электрических напряжений, возникающих на выводах его схемы, будет наиболее эффективным с точки зрения реализации сбоя (подобный эффект частично описан в [2]).

Было исследовано большое количество осциллограмм на выходе МК при разных напряженностях поля. Как и ожидали, импульс излучения случайным образом действовал в разные моменты времени: в фазе логической „единицы“ или „нуля“ или, что очень редко, в момент перехода из одного состояния в другое. Как правило, при действии эффективного импульса излучения в фазе „нуля“", происходил переход на высокий уровень и, наоборот, при действии в фазе „единицы“ происходил переход на низкий уровень.

Измерения произвольной выборки временных интервалов $\Delta t$ между моментом последнего (предсбойного) переключения с одного логического уровня на другой и моментом действия эффективного импульса излучения показали следующее. Величина $\Delta t$ составила $0.45-0.5 \mu \mathrm{s}$ при длительности половины периода меандра $0.62 \mu \mathrm{s}$, и она сопровождалась значительный 


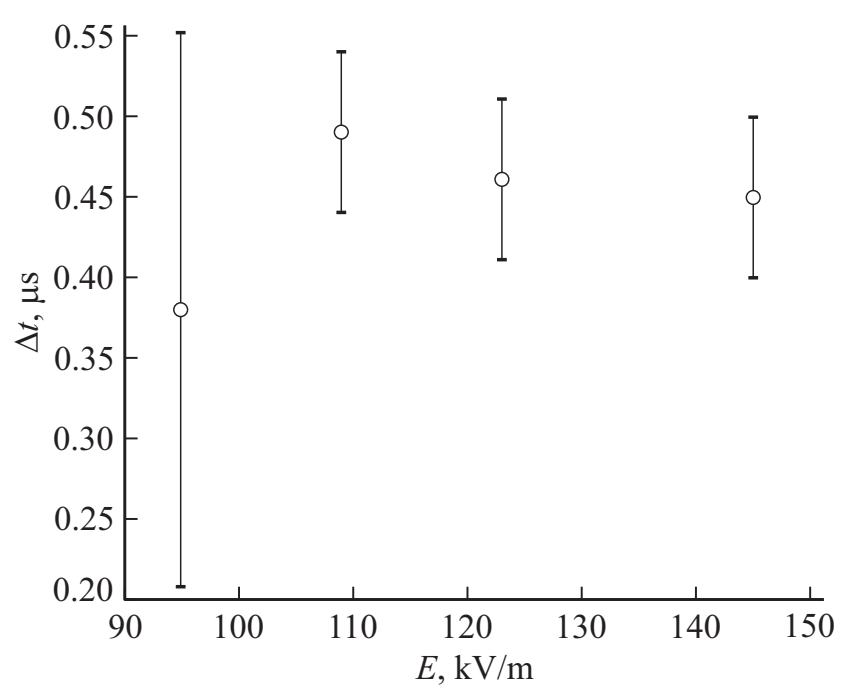

Рис. 4. Зависимость временной задержки $\Delta t$ между моментом действия СШП излучения и фазой генерации меандра, приводящей к сбою.

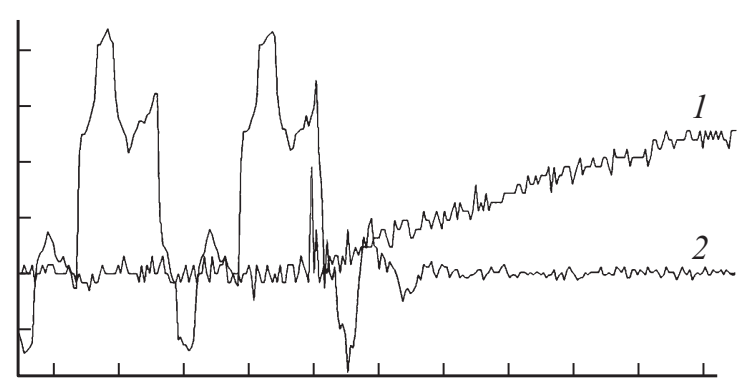

Рис. 5. Осциллограмма меандра (2) - реализация длительного сбоя с наложенным изображением интеграла действующего импульса излучения (1). Время - $500 \mathrm{~ns} / \mathrm{div}$. Амплитуда $0.5 \mathrm{~V} / \mathrm{div}$.

разбросом данных при низких уровнях напряженности поля (разброс посчитан на основе выборок из 20 сбоев для каждой серии) (рис. 4). Такой разброс явился следствием сложности точного измерения времени из-за помехового сигнала, наложенного на меандр (рис. 5).

\section{3. Действие тормозного излучения}

При исследованиях действия ТИ МК располагали напротив анода ускорительной трубки установки. Величину экспозиционной дозы облучения изменяли с использованием поглотителя из свинца разной толщины, который размещали перед МК, в некоторых случаях изменяли и расстояние до анода установки. Погрешность измерения экспозиционной дозы составляла $\pm 20 \%$.

На рис. 6 приведены осциллограммы сигнала с выхода МК и амплитудно-временного профиля ТИ, полученных в пусках при изменении дозы D и мощности дозы его облучения $P$.
Характерными особенностями осциллограмм сигналов на выходе МК (рис. 6) являются:

- при малой величине дозы ( единицы Röntgen) изменения длительности генерируемых фаз не отмечали, т.е. сбоя в работе МК нет;

- при увеличении дозы и действии ТИ в фазе „нуля“ (рис. 6, $a$ ) или „единицы“ (рис. 6, $b$ ) происходит увеличение длительности этой фазы - нарушение штатной работы.

В то же время случались события, когда изменялись длительности обеих фаз. Как видно из осциллограммы (рис. $6, c$ ), при величине дозы $24 \mathrm{R}$ излучение действовало в момент перехода МК из фазы генерации „единицы“ в фазу „нуля“, что привело к увеличению длительностей обеих фаз. Дальнейшее увеличение дозы ТИ в такой же момент его действия привело к реализации зависания (рис. 6, $d$ ).

Несмотря на небольшое количество данных для их статистической обработки (число пусков мощной установки ТИ всегда ограничено), можно проанализировать полученные результаты с точки зрения влияния на реакцию МК момента действия излучения. Как видно из рис. 6, наблюдали два вида эффектов: уширение генерируемых фаз сигналов, которое квалифицировали как сбой в работе, и зависание. Зависание - это наиболее радикальный эффект, и оно возникало при действии ТИ в следующие моменты времени:

- в момент переключения из „нуля“ в „единицу“ или наоборот;

— в момент генерации „единицы“ через $0.3-0.4 \mu \mathrm{s}$ от начала этой фазы;

- в момент генерации „нуля“ через $0.5 \mu \mathrm{s}$ от начала этой фазы.

Таким образом, видно, что существует определенная связь между генерируемой МК-фазой и его реакцией на облучение при зависании. В остальных случаях наблюдали уширение генерируемой фазы на величину от 0.15 до $1-1.1 \mu \mathrm{s}$.

Bсе наблюдаемые эффекты зависят и от величины дозы облучения МК. Так, до величины дозы $\sim 25 \mathrm{R}$ зависание не зарегистрировано, свыше 46 R, включая и это значение (в диапазоне доз 25-45 R данные не получены), зависание регистрировали во всех случаях.

Несмотря на единичные реализованные случаи, необходимо обратить особенное внимание на действие ТИ в моменты смены фаз с „нуля“ на „единицу“ и наоборот. Как было получено, это - наиболее важные фазы, с точки зрения влияния на реакцию МК. Так, облучение ТИ, момент действия которого совпал с переходом из „нуля“ в „единицу“, привело к реализации зависания при дозе 6R, что почти на порядок ниже, чем зарегистрировано в других случаях. В то же время, когда излучение действовало в момент перехода из „единицы“ в „ноль“, при дозе $82 \mathrm{R}$ реакция МК зарегистрирована не была. Отсюда видно, что момент действия излучения относительно фазы работы МК оказывает существенное влияние на его реакцию, что совпадает с результатами, 

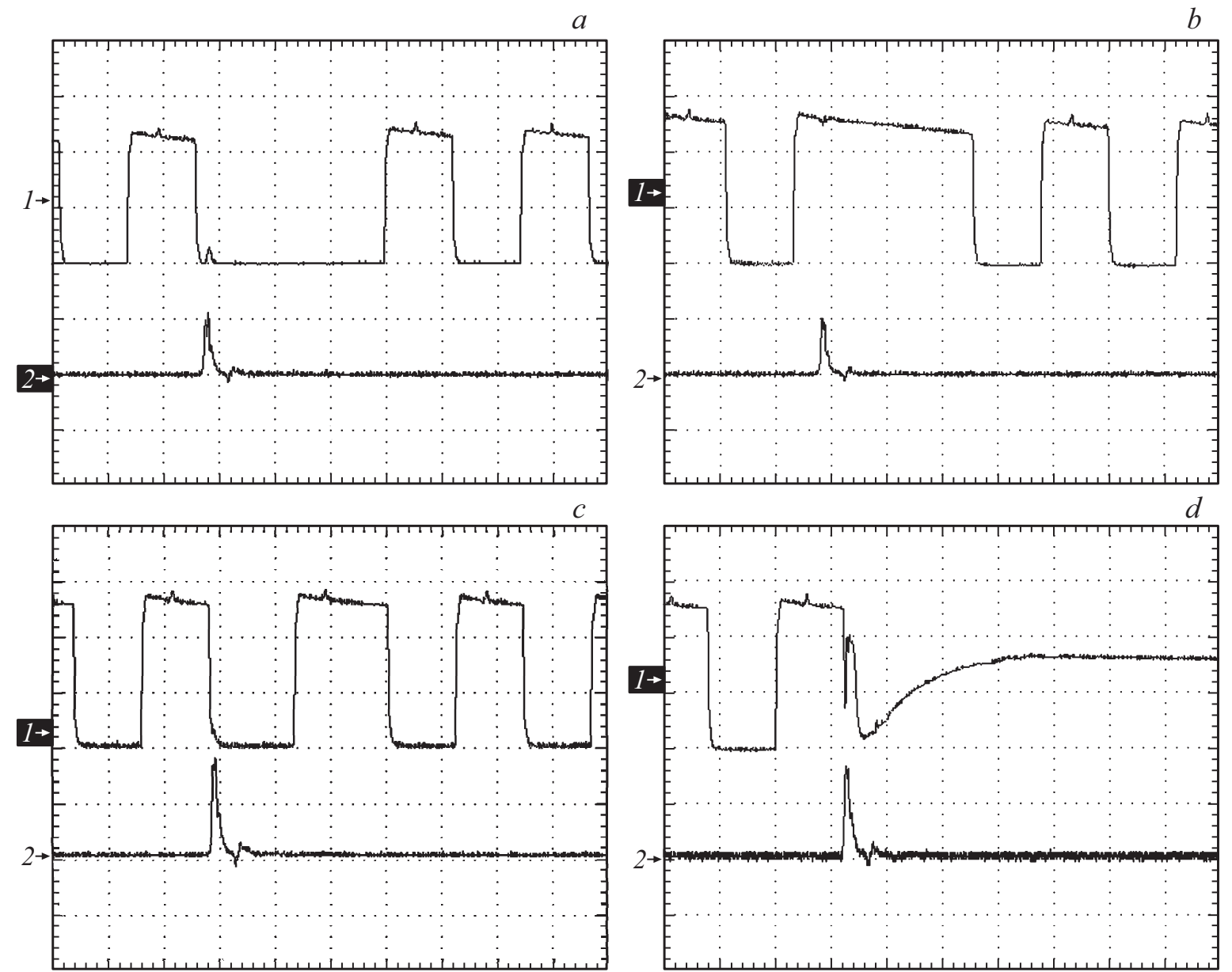

Рис. 6. Осциллограммы сигналов при действии ТИ. Верхний луч - сигнал с выхода МК $(1 \mathrm{~V} / \mathrm{div})$, нижний луч - временной профиль ТИ. $a-D=13 \mathrm{R}, P=2.6 \cdot 10^{8} \mathrm{R} / \mathrm{s} ; b-D=25 \mathrm{R}, P=5.6 \cdot 10^{8} \mathrm{R} / \mathrm{s} ; c-D=24 \mathrm{R}, P=5 \cdot 10^{8} \mathrm{R} / \mathrm{s} ; d-D=120 \mathrm{R}$, $P=2.7 \cdot 10^{9} \mathrm{R} / \mathrm{s}$. Время $-500 \mathrm{~ns} / \mathrm{div}$.

полученными при воздействии СШП-излучения. Таким образом, показано, что реакция МК на действие ТИ зависит и от величины дозы облучения, и от момента действия ТИ относительно фазы его работы.

\section{4. Действие импульсов электрического напряжения}

Для выяснения реакции МК на действие импульсов электрического напряжения с длительностью, близкой к длительности действия ТИ, были проведены исследования в лабораторных условиях при количестве повторений воздействия, равном 200; схема измерений представлена на рис. 7. Изменяли длительность импульса напряжения и его амплитуду. Импульс подавали в цепь питания МК, а для исключения его шунтирования конденсаторами источника питания использовали защиту в виде индуктивности обмотки $L$ импульсного трансформатора МИТ-12.

В табл. 1 и 2 приведены данные о реакции МК на действие импульсов. Указаны: амплитуда импуль- са $U(\mathrm{~V})$; вероятность сбоя или зависания (\%); промежуток времени между моментом смены фазы меандра и воздействием (от спада - переход из „единицы“ в „ноль““, от фронта - переход из „нуля““ в „единицу“, $\mu \mathrm{s})$; величина уширения генерируемой фазы ( $\mu \mathrm{s})$.

Из табл. 1 следует, что при длительности импульса 20 ns:

- при амплитуде напряжения $200 \mathrm{~V}$ реализуется только сбой;

- с увеличением амплитуды импульса увеличивается вероятность сбоя, и реализуются случаи зависания;

- вероятность сбоя выше, чем зависания.

Как следует так же из табл. 1 , сбой реализуется:

- при действии импульса напряжения через $0.3-0.45 \mu \mathrm{s}$ от начала генерации „нуля“, и это приводит к уширению фазы на половину периода меандра;

- при действии импульса напряжения в фазе генерации „единицы“ в большинстве случаев наблюдается уширение на 0.62 или $0.75 \mu$ s через $0.45 \mu$ s от фронта.

При длительности импульса $50 \mathrm{~ns}$ (табл. 2) вероятности реализации сбоя или зависания такого же уровня по величине и с теми же временными интервалами, 
Таблица 1. Параметры, характеризующие реакцию МК на действие импульсов напряжения длительностью $20 \mathrm{~ns}$ разной амплитуды

\begin{tabular}{|c|c|c|c|c|c|c|c|c|}
\hline \multirow{3}{*}{$\begin{array}{l}U \\
\mathrm{~V}\end{array}$} & \multicolumn{5}{|c|}{ Сбой } & \multicolumn{3}{|c|}{ Зависание } \\
\hline & \multirow{2}{*}{$\begin{array}{c}\text { Вероятность } \\
\text { сбоя, } \\
\%\end{array}$} & \multicolumn{2}{|c|}{ Генерация „нуля“ } & \multicolumn{2}{|c|}{ Генерация „единицы“ } & \multirow{2}{*}{$\begin{array}{c}\text { Вероятность } \\
\text { зависания, } \\
\%\end{array}$} & \multirow{2}{*}{$\begin{array}{c}\text { От спада, } \\
\mu \mathrm{s}\end{array}$} & \multirow{2}{*}{$\begin{array}{c}\text { От фронта, } \\
\mu \mathrm{s}\end{array}$} \\
\hline & & $\begin{array}{c}\text { От спада, } \\
\mu \mathrm{s}\end{array}$ & $\begin{array}{c}\text { Уширение } \\
\mu \mathrm{s}\end{array}$ & $\begin{array}{c}\text { От фронта, } \\
\mu \mathrm{s}\end{array}$ & $\begin{array}{c}\text { Уширение } \\
\mu \mathrm{s}\end{array}$ & & & \\
\hline 200 & 2 & 0.33 & 0.62 & 0.45 & $0.62 ; 0.75$ & 0 & - & - \\
\hline 212 & 3.5 & $0.3-0.4$ & 0.62 & 0.45 & 0.75 & 3.5 & 0.55 & 0.56 \\
\hline 300 & 8 & $0.3-0.45$ & 0.62 & $0.3-0.45$ & 0.62 & 5 & 0.55 & 0.57 \\
\hline
\end{tabular}

Таблица 2. Параметры, характеризующие реакцию МК на действие импульсов напряжения длительностью $50 \mathrm{~ns}$ разной амплитуды

\begin{tabular}{|c|c|c|c|c|c|c|c|c|}
\hline \multirow{3}{*}{$\begin{array}{l}U \\
\mathrm{~V}\end{array}$} & \multicolumn{5}{|c|}{ Сбой } & \multicolumn{3}{|c|}{ Зависание } \\
\hline & \multirow{2}{*}{$\begin{array}{c}\text { Вероятность } \\
\text { сбоя, } \\
\%\end{array}$} & \multicolumn{2}{|c|}{ Генерация „нуля“ } & \multicolumn{2}{|c|}{ Генерация „единицы“ } & \multirow{2}{*}{$\begin{array}{c}\text { Вероятность } \\
\text { зависания, } \\
\%\end{array}$} & \multirow{2}{*}{$\begin{array}{c}\text { От спада, } \\
\mu \mathrm{s}\end{array}$} & \multirow{2}{*}{$\begin{array}{c}\text { От фронта, } \\
\mu \mathrm{s}\end{array}$} \\
\hline & & $\begin{array}{c}\text { От спада, } \\
\mu \mathrm{s}\end{array}$ & $\begin{array}{c}\text { Уширение } \\
\mu \mathrm{s}\end{array}$ & $\begin{array}{c}\text { От фронта, } \\
\mu \mathrm{s}\end{array}$ & $\begin{array}{c}\text { Уширение, } \\
\mu \mathrm{s}\end{array}$ & & & \\
\hline $\begin{array}{c}25 \\
0\end{array}$ & 2.5 & $0.3-0.42$ & 0.62 & $0.3-0.45$ & $0.62-0.68$ & 5.5 & 0.55 & $0.55-0.6$ \\
\hline $\begin{array}{c}27 \\
0\end{array}$ & 6 & $0.3-0.42$ & $0.63-0.75$ & $0.3-0.42$ & $0.63-0.75$ & 4.5 & 0.53 & 0.55 \\
\hline
\end{tabular}

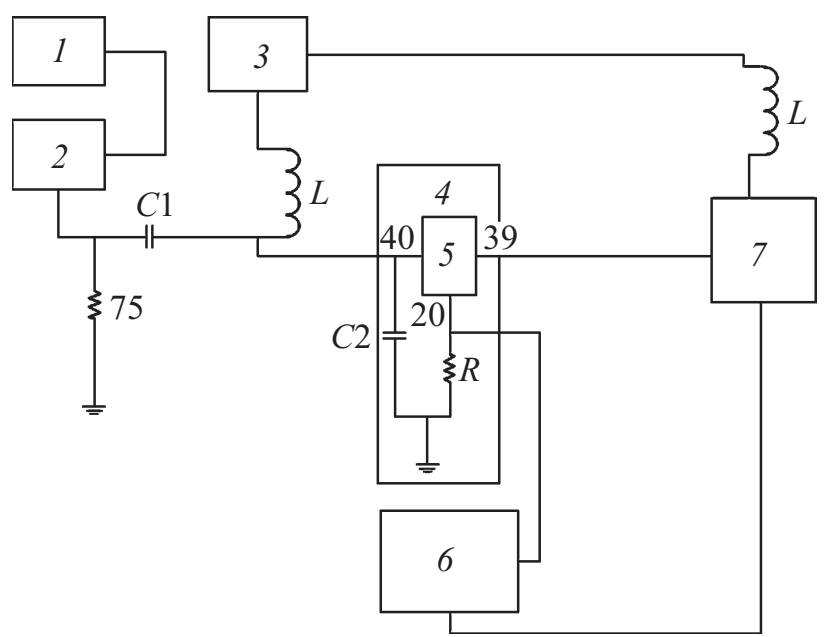

Рис. 7. Электрическая схема измерений: 1 - генератор запуска Г5-54, 2 - генератор импульсов напряжения Г5-13, 3 - источник питания Б5-7, 4 - корпус для размещения МК, 5 - МК, 6 - цифровой осциллограф TDS 2014, 7 - эмиттерный повторитель, $R$ - резистор нагрузки МК, $L$ - обмотка импульсного трансформатора МИТ-12.

т. е. существенные отличия во временных зависимостях отсутствуют. Тем не менее эти измерения необходимо было провести, поскольку в этом диапазоне времен изменялась и длительность ТИ.

\section{5. Обсуждение результатов}

Из приведенных результатов видно, что при действии импульса электрического напряжения или при облучении ТИ или при действии СШП ЭМИ реакция МК близкая: наблюдается сбой в работе (уширение генерируемой фазы меандра) или зависание. Более того, временные интервалы между моментом смены фаз меандра и действием этих помеховых факторов $(\Delta t)$, при которых наблюдаются эффекты их воздействия, во всех случаях достаточно близки: $0.3-0.45 \mu \mathrm{s}-$ для электрического импульса; $0.3-0.5 \mu \mathrm{s}$ - для ТИ; $0.45-0.5 \mu \mathrm{s}-$ для СШП ЭМИ.

Как следует из табл. 1 и 2, при действии импульса напряжения генератора зависание реализуется через $\sim 0.55 \mu$ s от момента перехода генерируемой „единицы“ в „ноль“, или через это же время от перехода „нуля“ в „единицу“, т.е. в непосредственной близости к смене фазы генерации меандра (за $\sim 0.07 \mu$ s до очередной смены при длительности половины периода генерации $\sim 0.62 \mu \mathrm{s})$. Анализ, проведенный на основе результатов, полученных при действии СШП ЭМИ, показал, что зависание реализуется при практически таком же временном интервале между моментом смены фаз в меандре и моментом действия излучения, так же, как и при действии ТИ. Таким образом, можно констатировать близость моментов действия всех помеховых факторов 
относительно фазы генерации меандра МК, приводящих к нарушению его работы, заложенной в программу.

Тем не менее существуют и различия между реакциями МК на действие этих факторов. В случае действия однократных электрических импульсов или одиночного импульса ТИ уширение генерируемой фазы меандра составляет величину, близкую к половине его периода для электрических импульсов $(0.62-0.75 \mu \mathrm{s})$ или находится в диапазоне $0.15-1.1 \mu \mathrm{s}$ - при действии ТИ. К сожалению, реально возможное относительно небольшое количество пусков установки ТИ не позволило оценить эту величину более точно. Можно только предположить, что уширение зависит от дозы (мощности дозы) облучения ТИ.

Как указано выше, в случае СШП ЭМИ исследования были проведены при действии пачек импульсов. Существование различных комбинаций между моментом действия очередного импульса пачки и генерируемыми импульсами меандра, при наличии эффекта восстановления генерации одним из импульсов излучения, приводит к сложной связи между импульсами пачки и длительностью сбоя в работе МК. Она изменяется в пределах от $\delta$-сбоя до нескольких десятков ms. Поэтому сравнение длительности такого сбоя с уширением при действии электрического импульса или ТИ становится некорректным.

Тем не менее можно предложить следующий механизм воздействия, который приводит к сбою в работе МК во всех исследуемых ситуациях, а именно изменение режима работы его электрической схемы. Так, при действии короткого (десятки ns) импульса электрического напряжения с амплитудой, много большей напряжения питания $5 \mathrm{~V}$, изменяется величина напряжения питания, в результате чего некоторые компоненты схемы могут оказаться в насыщении, что приводит к появлению интервала времени микросекундного диапазона, требуемого для восстановления штатного режима работы МК. Амплитуда „порогового“ напряжения составляет около $200 \mathrm{~V}$. Превышение этого значения приводит к реализации зависания. Здесь отметим, что, как и в случае СШП ЭМИ, существенна пороговость значения этой амплитуды.

При действии СШП ЭМИ источником паразитных напряжений и токов являют- ся элементы многослойной разводки МК. Скорость стекания паразитного заряда напрямую зависит от текущего электрического режима активного элемента схемы, к которому подключен элемент разводки. Поэтому время релаксации может варьировать в значительных пределах.

При облучении ТИ изменение режима работы достигается за счет возникающей проводимости кристалла полупроводника: при его ионизации в электрической схеме появляются дополнительные носители тока. Время их релаксации и восстановления работы схемы при сбое определяется временем жизни носителей заряда в кремнии и не зависит от режима работы прибора. Оно не превышает периода меандра, что близко к реакции
МК на действие импульса электрического напряжения с длительностью ТИ.

Была проведена так же оценка радиационной стойкости МК типа ATmega8515. Суммирование доз ТИ в пусках установки, в которых исследовали его реакцию на воздействие, показало, что была достигнута доза облучения МК около $5 \mathrm{kR}$ (доза изменялась в пусках от 6 до 800 R), что ниже величины, приведенной в [5]. Для уточнения допустимой дозы, при которой по использованной методике контроля еще не фиксируется необратимое нарушение работоспособности МК (статический ток потребления не увеличивается), были проведены дополнительные пуски установки. В конечном итоге величина достигнутой дозы составила свыше $24 \mathrm{kR}$, и необратимые изменения не отмечены.

После набранной этой величины дозы было проведено перепрограммирование МК с использованием внутренней флэш-памяти, которое подтвердило сохранение его работоспособности. В [5] отказ флэш-памяти в МК наблюдали при дозе 4.7 и $9.5 \mathrm{kR}$ (для разных образцов), облученных в пассивном режиме, и при 11 и $13 \mathrm{kR}$ (для разных образцов) при облучении в активном режиме. Это позволяет утверждать, что при импульсном облучении МК данного типа (диапазон изменения величины дозы во всех пусках составил от 6 до $5400 \mathrm{R}$ ) его радиационная стойкость значительно выше, чем при стационарном.

\section{Заключение}

Впервые исследована в динамическом режиме одна из сложно-функциональных интегральных микросхем МК на раздельное воздействие ТИ и СШП ЭМИ. В числе наиболее интересных и значимых итогов получено, что, несмотря на зависимость результатов действия ТИ от дозы облучения МК, его реакция на действие ионизации близка к реакции на действие СШП ЭМИ.

Наиболее критичным моментом действия излучений является смена генерируемых фаз меандра, т.е. совпадение момента действия излучений с фронтом или спадом генерируемой фазы. Наличие этого эффекта зарегистрировано и в условиях действия импульсного напряжения в цепь питания МК. Отличительной чертой этих экспериментов было то, что, как правило, величина уширения фазы при сбое была близка к половине периода генерируемого меандра.

Предложен механизм воздействия исследованных помеховых факторов на функционирование МК, который приводит к сбою в его работе, а именно изменение режима работы его электрической схемы.

Полученные данные позволяют также утверждать, что при импульсном облучении МК данного типа его радиационная стойкость значительно выше, чем при стационарном облучении: величина достигнутой дозы без проявления необратимых нарушений в работоспособности составила свыше $24 \mathrm{kR}$, т.е. получено превышение дозы свыше двух раз по сравнению с результатами [5]. 


\section{Список литературы}

[1] Camp M., Gerth H., Garbe H., Haas H. // IEEE Trans. on EMC. 2004. Vol. 46. N 3. P. 368-379.

[2] Clarke T., French D. // Proceeding of ICEAA Conference, Torino, Italy, 2012. D04X.

[3] French D.M., Clarke T. // AMEREM-2014. Book of Abstracts. ID096. University of Albuquerque, New Mexico, USA. July 27-31. 2014.

[4] Степовик А.П., Шамаев Е.Ю., Арманов М.М. // Радиотехника и электроника. 2017. Т. 62. № 8. С. 812-818.

[5] Лоскутов И.О., Калашников А.А., Некрасов П.В. // 20-я научно-техническая конф. „Радиационная стойкость электронных систем - СТОЙКОСТЬ-2017“. Лыткарино, 6-7 июня 2017. Тезисы докладов. 2017. С. 196-197.

[6] Лаврентьев Б.Н., Мунасыпов Р.Н., Павловских Д.Е. и др. // Вопросы атомной науки и техники. Сер.: Физика радиационного воздействия на радиоэлектронную аппаратуру. 2012. Вып. 2. С. 105-109.

[7] Лазарев Ю.Н., Петров П.В. // ЖЭТФ. 1999. Т. 115. Вып. 5. C. $1689-1707$.

[8] Кондратьев А.А., Лазарев Ю.Н., Потапов А.В. и др. // ДАН. 2011. Т. 438. № 5. С. 615-618.

[9] Сахаров К.Ю. Излучатели сверхкоротких электромагнитных импульсов и методы измерения их параметров. М.: Московский гос. ин-т электроники и математики, 2006. $159 \mathrm{c}$. 